\title{
CARMENES: An Overview Six Months After First Light
}

A. Quirrenbach ${ }^{\mathrm{a}}$, P. J. Amado ${ }^{\mathrm{b}}$, J. A. Caballero ${ }^{\mathrm{a}, \mathrm{c}}$, R. Mundt ${ }^{\mathrm{d}}$, A. Reiners $^{\mathrm{e}}, \mathrm{I}_{\text {. Ribas }}^{\mathrm{f}}$, W. Seifert ${ }^{\mathrm{a}}$, M. Abril ${ }^{\mathrm{b}}$, J. Aceituno ${ }^{\mathrm{g}}$, F. J. Alonso-Floriano ${ }^{\mathrm{h}}$, H. Anwand-Heerwart ${ }^{\mathrm{e}}$, M. Azzaro ${ }^{g}$, F. Bauer ${ }^{\mathrm{e}}$, D. Barrado ${ }^{\mathrm{c}}$, S. Becerril ${ }^{\mathrm{b}}$, V. J. S. Béjar ${ }^{\mathrm{i}}$, D. Benítez ${ }^{\mathrm{g}}$, Z. M. Berdiñas ${ }^{\mathrm{b}}$, M. Brinkmöller ${ }^{\mathrm{a}}$, M. C. Cárdenas ${ }^{\mathrm{d}}$, E. Casal $^{\mathrm{b}}$, A. Claret $^{\mathrm{b}}$, J. Coloméf ${ }^{\mathrm{f}}$ M. Cortés-Contreras ${ }^{\mathrm{h}}$,

S. Czesla ${ }^{j}$, M. Doellinger ${ }^{k}$, S. Dreizler ${ }^{\mathrm{e}}$, C. Feiz ${ }^{\mathrm{a}}$, M. Fernández ${ }^{\mathrm{b}}$, I. M. Ferro ${ }^{\mathrm{b}}$, B. Fuhrmeister ${ }^{\mathrm{j}}$, D. Galadíg, I. Gallardoh ${ }^{\mathrm{h}}$ M. C. Gálvez-Ortiz ${ }^{\mathrm{c}}$, Á. García-Piquer $^{\mathrm{f}}$, R. Garrido ${ }^{\mathrm{b}}$, L. Gesa $^{\mathrm{f}}$, V. Gómez Galerag , J. I. González Hernándezi , R. González Peinado ${ }^{\mathrm{h}}$, U. Grözinger ${ }^{\mathrm{d}}$, J. Guàrdiaf ${ }^{f}$ E. W. Guenther ${ }^{k}$, E. de Guindos ${ }^{g}$, H.-J. Hagen $^{j}$, A. P. Hatzes ${ }^{k}$, P. H. Hauschildt ${ }^{j}$, J. Helmlings ${ }^{g}$, T. Henningd ${ }^{d}$, D. Hermann ${ }^{\mathrm{d}}$, R. Hernández Arabíg ${ }^{\mathrm{g}}$, L. Hernández Castañog ${ }^{\mathrm{g}}$,

F. Hernández Hernandog ${ }^{\text {, E. Herrero }}{ }^{f}$, A. Huber ${ }^{\mathrm{d}}$, K. F. Huber ${ }^{\mathrm{j}}$, P. Huke ${ }^{\mathrm{e}}$, S. V. Jeffers ${ }^{\mathrm{e}}$,

E. de Juan' ${ }^{\mathrm{g}}$, A. Kaminski ${ }^{\mathrm{a}}$, M. Kehr ${ }^{\mathrm{k}}$, M. Kimª ${ }^{\mathrm{a}}$, R. Klein ${ }^{\mathrm{d}}$, J. Klüter ${ }^{\mathrm{a}}$, M. Kürster ${ }^{\mathrm{d}}$, M. Lafarga ${ }^{f}$, L. M. Lara ${ }^{\mathrm{b}}$, A. Lamert ${ }^{\mathrm{e}}$, W. Laun ${ }^{\mathrm{d}}$, R. Launhardt ${ }^{\mathrm{d}}$, U. Lemke ${ }^{\mathrm{e}}$, R. Lenzen ${ }^{\mathrm{d}}$, M. Llamas ${ }^{\mathrm{h}}$, M. López del Fresno ${ }^{\mathrm{c}}$, M. López-Puertas ${ }^{\mathrm{b}}$, J. López-Santiago ${ }^{\mathrm{h}}$, J. F. López Salas ${ }^{\mathrm{g}}$, H. Magán Madinabeitia ${ }^{\mathrm{b}}$, U. Mall ${ }^{\mathrm{d}}$, H. Mandel ${ }^{\mathrm{a}}$, L. Mancini ${ }^{\mathrm{d}}$, J. A. Marín Molina ${ }^{\mathrm{g}}$, D. Maroto Fernández ${ }^{\mathrm{g}}$, E. L. Martínc, S. Martín-Ruiz ${ }^{\mathrm{b}}$, C. Marvine ${ }^{\mathrm{e}}$, R. J. Mathar ${ }^{\mathrm{d}}$, E. Mirabet ${ }^{\mathrm{b}}$, D. Montes ${ }^{h}$, J. C. Morales ${ }^{\mathrm{f}}$, R. Morales Muñoz ${ }^{\mathrm{b}}$, E. Nagel ${ }^{\mathrm{j}}$, V. Naranjo ${ }^{\mathrm{d}}$, G. Nowak ${ }^{i}$ E. Palléi , J. Panduro ${ }^{\mathrm{d}}$, V. M. Passeggere, A. Pavlov ${ }^{\mathrm{d}}$, S. Pedraz ${ }^{\mathrm{g}}$, E. Pérez ${ }^{\mathrm{b}}$,

D. Pérez-Medialdea ${ }^{b}$, M. Perger ${ }^{f}$, M. Pluto ${ }^{k}$, A. Ramón ${ }^{b}$, R. Rebolo ${ }^{i}$, P. Redondo ${ }^{i}$, S. Reffert $^{\mathrm{a}}$,

S. Reinhart ${ }^{\mathrm{g}}$, P. Rhode ${ }^{\mathrm{e}}$, H.-W. Rix ${ }^{\mathrm{d}}$, F. Rodler ${ }^{\mathrm{d}}$, E. Rodríguez ${ }^{\mathrm{b}}$, C. Rodríguez López ${ }^{\mathrm{b}}$,

R.-R. Rohloff ${ }^{\mathrm{d}}$, A. Rosich ${ }^{\mathrm{f}}$, M. A. Sánchez Carrasco ${ }^{\mathrm{b}}$, J. Sanz-Forcada ${ }^{\mathrm{c}}$, P. Sarkis ${ }^{\mathrm{d}}$,

L. F. Sarmiento ${ }^{\text {e, S. Schäfer }}{ }^{\mathrm{e}}$, J. Schiller ${ }^{\mathrm{k}}$, C. Schmidt ${ }^{\mathrm{e}}$, J. H. M. M. Schmitt ${ }^{\mathrm{j}}$, P. Schöfer ${ }^{\mathrm{e}}$,

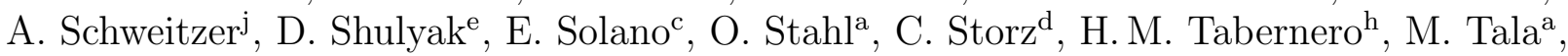

L. Tal-Or ${ }^{\mathrm{e}}$, R.-G. Ulbrich ${ }^{\mathrm{e}}$, G. Veredas ${ }^{\mathrm{a}}$, J. I. Vico Linares ${ }^{\mathrm{g}}$, F. Vilardell ${ }^{\mathrm{f}}, \mathrm{K}$. Wagner ${ }^{\mathrm{a}}$,

J. Winklerk ${ }^{\mathrm{k}}$, M. R. Zapatero Osorio ${ }^{\mathrm{c}}$, M. Zechmeister ${ }^{\mathrm{e}}$, M. Ammler-von Eiff ${ }^{\mathrm{l}}$,

G. Anglada-Escudém ${ }^{m}$ C. del Burgon ${ }^{n}$, M. L. García-Vargas ${ }^{o}$, A. Klutsch ${ }^{\mathrm{q}}$, J.-L. Lizon ${ }^{\mathrm{r}}$, M. López-Morales ${ }^{\mathrm{s}}$, A. Ofir ${ }^{\mathrm{t}, \mathrm{e}}$, A. Pérez-Calpena ${ }^{\mathrm{o}}$, M. A. C. Perryman ${ }^{\mathrm{u}}$, E. Sánchez-Blanco ${ }^{\mathrm{v}}$,

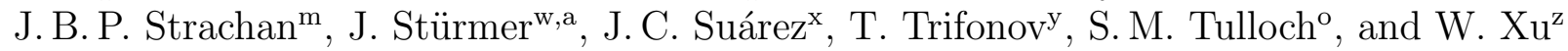

aLandessternwarte, Zentrum für Astronomie der Universität Heidelberg, Königstuhl 12, D-69117 Heidelberg, Germany

bInstituto de Astrofísica de Andalucía (CSIC), Glorieta de la Astronomía s/n, E-18008 Granada, Spain

${ }^{\mathrm{c}}$ Centro de Astrobiología (CSIC-INTA), Campus ESAC, Camino Bajo del Castillo s/n, E-28692 Villanueva de la Cañada, Madrid, Spain

dMax-Planck-Institut für Astronomie, Königstuhl 17, D-69117 Heidelberg, Germany

eInstitut für Astrophysik, Georg-August-Universität Göttingen, Friedrich-Hund-Platz 1, D-37077 Göttingen, Germany

fInstitut de Ciències de l'Espai (CSIC-IEEC), Campus UAB, c/ de Can Magrans s/n, E-08193 Bellaterra, Barcelona, Spain

${ }^{\text {g} C e n t r o ~ A s t r o n o ́ m i c o ~ H i s p a n o-A l e m a ́ n ~(M P G-C S I C), ~ O b s e r v a t o r i o ~ A s t r o n o ́ m i c o ~ d e ~ C a l a r ~ A l t o, ~}$ Sierra de los Filabres, E-04550 Gérgal, Almería, Spain

h Departamento de Astrofísica, Facultad de Física, Universidad Complutense de Madrid,

E-28040 Madrid, Spain

Ground-based and Airborne Instrumentation for Astronomy VI, edited by Christopher J. Evans, Luc Simard, Hideki Takami Proc. of SPIE Vol. 9908, 990812 - @ 2016 SPIE · CCC code: 0277-786X/16/\$18 · doi: 10.1117/12.2231880 
instituto de Astrofísica de Canarias, c/ Vía Láctea s/n, E-38205 La Laguna, Tenerife, Spain \&

Departamento de Astrofísica, Universidad de La Laguna, E-38206 Tenerife, Spain

${ }^{j}$ Hamburger Sternwarte, Gojenbergsweg 112, D-21029 Hamburg, Germany

kThüringer Landessternwarte Tautenburg, Sternwarte 5, D-07778 Tautenburg, Germany

${ }^{1}$ Max-Planck-Institut für Sonnensystemforschung, Justus-von-Liebig-Weg 3, D-37077

Göttingen, Germany

${ }^{\mathrm{m}}$ School of Physics and Astronomy, Queen Mary, University of London, 327 Mile End Road, London, E1 4NS, UK

${ }^{\mathrm{n}}$ Instituto Nacional de Astrofísica, Óptica y Electrónica, Luis Enrique Erro 1, Tonantzintla, 72840 Puebla, Mexico

oFRACTAL SLNE, c/ Tulipán 2, p. 13, 1A, E-28231 Las Rozas, Madrid, Spain

IINAF - Osservatorio Astrofisico di Catania, via S. Sofia 78, 95123 Catania, Italy

${ }^{\mathrm{r}}$ European Organisation for Astronomical Research in the Southern Hemisphere,

Karl-Schwarzschild-Str. 2, D-85748 Garching bei München, Germany

sHarvard-Smithsonian Center for Astrophysics, 60 Garden Street, Cambridge, MA 02138, USA

${ }^{t}$ Department of Earth and Planetary Sciences, Weizmann Institute of Science, 234 Herzl

Street, 76100 Rehovot, Israel

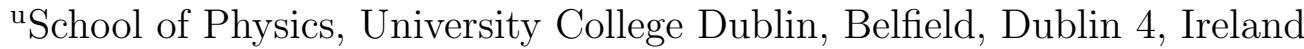

${ }^{v}$ Optical Development, Avenida Pablo Iglesias 7, local 5, E-41008 Sevilla, Spain

${ }^{w}$ Department of Astronomy and Astrophysics, University of Chicago, 5640 S. Ellis Avenue, 60637 Chicago, IL, USA

xUniversidad de Granada, Departamento de Física Teórica y de Cosmos, Fuentenueva

Campus, E-18007, Granada, Spain

${ }^{y}$ Department of Earth Sciences, James Lee Building, The University of Hong Kong, Pokfulam

Road, Hong Kong

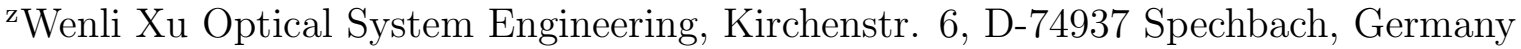

\begin{abstract}
The CARMENES instrument is a pair of high-resolution $(R \gtrsim 80,000)$ spectrographs covering the wavelength range from 0.52 to $1.71 \mu \mathrm{m}$, optimized for precise radial velocity measurements. It was installed and commissioned at the $3.5 \mathrm{~m}$ telescope of the Calar Alto observatory in Southern Spain in 2015. The first large science program of CARMENES is a survey of $\sim 300 \mathrm{M}$ dwarfs, which started on Jan 1, 2016.

We present an overview of all subsystems of CARMENES (front end, fiber system, visible-light spectrograph, near-infrared spectrograph, calibration units, etalons, facility control, interlock system, instrument control system, data reduction pipeline, data flow, and archive), and give an overview of the assembly, integration, verification, and commissioning phases of the project. We show initial results and discuss further plans for the scientific use of CARMENES.
\end{abstract}

Keywords: Spectrographs, Optical Instrumentation, Near-Infrared Instrumentation, Extrasolar Planets, M Dwarfs

\title{
1. INTRODUCTION: THE CARMENES PROJECT
}

CARMENES (Calar Alto high-Resolution search for $\mathbf{M}$ dwarfs with Exo-earths with Near-infrared and optical Echelle Spectrographs) is a key project at the $3.5 \mathrm{~m}$ telescope of the Calar Alto Observatory (CAHA) in the province of Andalucía in Southern Spain. It comprises two phases: the construction of a pair of high-resolution

Further author information: A.Quirrenbach@lsw.uni-heidelberg.de 
spectrographs covering the wavelength range from 0.52 to $1.71 \mu \mathrm{m}$, and a subsequent radial-velocity survey of $\sim 300 \mathrm{M}$ dwarfs with the aim of detecting terrestrial planets in their habitable zones. Both project phases are carried out by a consortium consisting of eleven institutions in Germany and Spain (institutions ${ }^{\mathrm{a}}$ through ${ }^{\mathrm{k}}$ of the author list of this paper).

In the course of the years 2014 and 2015, all subsystems of CARMENES were pre-integrated and tested at the premises of CARMENES partners, then moved to CAHA, and finally integrated with each other and the telescope. This process culminated in "First Light" of the complete instrument - defined as taking stellar spectra with both spectrographs simultaneously - on Nov 9, 2015. The infrared arm of CARMENES is thus the first on sky of a new generation of near-IR spectrographs optimized for precise radial-velocity work (see overview in Fischer et al. 2016).

This paper gives an overview of the CARMENES instrument as installed at the telescope, and discusses a few selected first results. Previous reports on the motivation for building CARMENES, and about progress on the design and construction of the instrument, were given by Quirrenbach et al. (2010, 2012, 2014). More details about individual subsystems of CARMENES can be found in the contributions by Becerril et al. (2016a,b), Caballero et al. (2016), Colomé et al. (2016), García-Vargas et al. (2016), Helmling et al. (2016), Pérez-Calpena et al. (2016), and Seifert et al. (2016) in these proceedings.

\section{INSTRUMENT SUB-SYSTEMS}

\subsection{Front End}

CARMENES receives the light of the CAHA $3.5 \mathrm{~m}$ telescope at the Cassegrain focus. A pick-up system including all optics is mounted in an adapter replacing the "standard" CAHA Cassegrain adapter ring, but with the same dimensions. This allows for a co-existence of the integral-field spectrograph PMAS (Roth et al. 2005) and CARMENES at the same telescope focus. Changing from one instrument to the other is simply done by remotely moving the pick-up mirror in or out of the beam.

The optical design of the front end consists of a flat $45^{\circ}$ pick-up mirror followed by an atmospheric dispersion compensator (ADC). The light coming from the telescope is then divided by a dichroic mirror at $0.96 \mu \mathrm{m}$ to feed the science fibers for the VIS and - via an additional fold mirror - the NIR spectrograph. The guide camera uses the light reflected back from a mirror in front of the VIS fiber head. A three-dimensional drawing of the front end is depicted in Colomé et al. (2016).

The ADC has been realized by a two double-prisms design to provide excellent correction of the atmospheric dispersion. The double-prisms are made of OHARA glasses S-BAL12 and SFTM16, which have very good internal transmission over the whole wavelength range. The residual dispersion remains below $5 \mu \mathrm{m}$ over the whole operational range to a maximum zenith distance of $70^{\circ}$. The two double prisms are counter-rotated for each observation to align the ADC with respect to the horizon.

A Starlight Xpress SVX-H16 CCD camera is used for acquisition and guiding. It looks at a mirror in front of the VIS fiber head which has two holes for the science and the calibration fiber separated by $88^{\prime \prime}$ with a diameter of $1^{\prime \prime} .5$. The camera provides a field of view with a diameter of $3^{\prime}$. Binning $2 \times 2$ pixel yields a pixel size of 0 '! 18 and read-out times of a few milliseconds.

\subsection{Fiber System}

CARMENES uses 14 fibers in total for different purposes (for details see Stürmer et al. 2014). For each channel large-core fibers $(D=400 \mu \mathrm{m})$ are used to couple the two calibration units to the front end at the telescope. For each of the two wavelength ranges, the starlight as well as the calibration light is fed in the front end into two $100 \mu \mathrm{m}$ circular fibers with a length of $42 \mathrm{~m}$ (VIS) / $50 \mathrm{~m}$ (NIR) each. For both wavelength channels, the same type of fiber with a low concentration of hydroxyl groups is used (CeramOptec WF), which provides high internal throughput within the whole spectral range of both instruments. Before entering the vacuum tank of each spectrograph, the circular fibers are linked to $\sim 8 \mathrm{~m}$ long fibers with a $100 \mu \mathrm{m}$ octagonal core by an FC-FC junction. This combination ensures very good scrambling of the fiber input, isolating the spectrographs from guiding errors and variations of the seeing. The fibers are continuously agitated to reduce modal noise. 

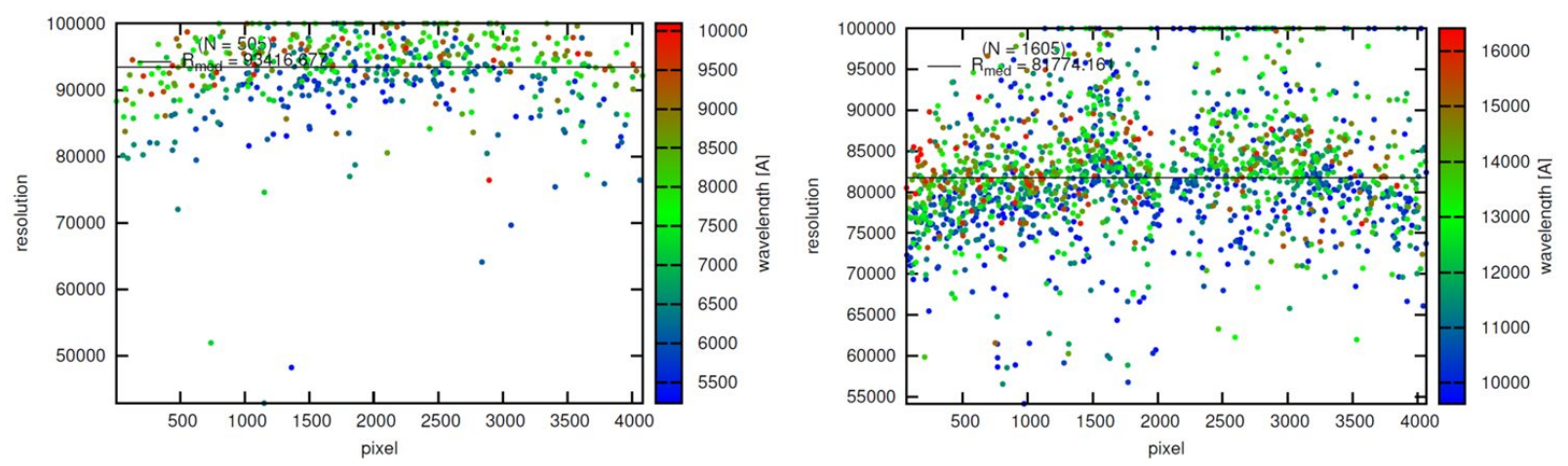

Figure 1. Spectral resolution of the VIS (left) and NIR (right) spectrographs of CARMENES, as measured with unresolved lines of a hollow-cathode lamp. The $x$ axis is the position on the detector in the main dispersion direction. The color scale encodes the wavelength.

For each channel an additional large-core fiber is used to guide light collected from the $0^{\text {th }}$ order of the echelle grating towards a photon counter (located in a separate rack) to provide information on the photonweighted midpoint of the exposure, which is needed for a precise correction of the observed radial velocities to the barycenter of the Solar System.

\subsection{Visible-Light Spectrograph}

The visible-light spectrograph of CARMENES covers the wavelength range of 0.52 to $0.96 \mu \mathrm{m}$ with a measured spectral resolution $R=93,400$ (see Fig. 1). The optical design of the instrument is a grism cross-dispersed, white-pupil, echelle spectrograph working in quasi-Littrow mode using a two-beam, two-slice, image slicer. It is housed in a thermally stabilized vacuum vessel at a pressure of $\sim 10^{-5}$ mbar and at a temperature of $\sim 12^{\circ} \mathrm{C}$. The detector is a $4096 \times 4096$ pixel CCD e2v 231-84. The design, construction, installation, and initial operation of the visible-light spectrograph are described in detail in Seifert et al. $(2012,2016)$.

\subsection{Near-IR Spectrograph}

The design of the near-IR spectrograph is very similar to that of its visible-light counterpart. The main difference (apart from the detector system) is its operating temperature $(\sim 140 \mathrm{~K})$, which necessitates a sophisticated cooling system (Becerril et al. 2016b). The NIR spectrograph covers the wavelength range from 0.96 to $1.71 \mu \mathrm{m}$ at a measured resolution $R=81,800$ (see Fig. 1). It employs two $2048 \times 2048$ pixel HAWAII-2RG infrared detectors with $2.5 \mu \mathrm{m}$ wavelength cutoff. The integration and performance testing of the NIR spectrograph under time pressure was among the most challenging tasks of the CARMENES project (Becerril et al. 2016a).

\subsection{Calibration Units and Etalons}

The calibration system of CARMENES consists of two calibration units and two temperature-stabilized FabryPérot etalons (one for the VIS and one for the NIR in each case), linked by fibers to the front end. Each calibration unit houses a quartz lamp for flat-fielding and up to seven hollow-cathode lamps for wavelength calibration; currently Th-Ne, U-Ne, and U-Ar lamps are in use. It is possible to switch on several lamps simultaneously, as a light-tight mechanism with a rotating mirror is used to select an "active lamp" for a specific exposure (see Quirrenbach et al. 2012, 2014 for details of the design). This provides maximum flexibility and allows for cross-calibration of multiple lamps.

The Fabry-Pérot units are also connected to the calibration units. They provide a regular comb of $\sim 15,000$ lines over the wavelength range of each spectrograph, which is far better for determining wavelength solutions and monitoring short-term radial-velocity drifts than the irregular spectrum of an emission line lamp. However, the lamps provide absolute wavelength calibration and better long-term stability; it is thus necessary to tie the etalons to them taking advantage of the capabilities of the calibration unit. 


\subsection{Facility Control and Interlock System}

An intelligent interlock system has been implemented for the control of CARMENES and its associated facilities (Helmling et al. 2016). It monitors the status of the instrument and of the auxiliary systems, and organizes information about their overall status and health. For safety reasons, it is essential to have an intelligent interlock system that informs the operator or observatory staff in case of a malfunction in any part of the instrument. To insure the safety of the instrument, the interlock system can also react autonomously to certain conditions, automatically starting vacuum pumps and opening or closing valves. This system receives inputs from all the critical elements and activates warnings and alarms in case of any abnormal function.

To protect all instrument subsystems, it is necessary to have well defined procedures for cool down, warm-up or re-pressurization of the tanks and cryostats. The interlock system ensures that these procedures are followed, since all interlock conditions have to be fulfilled before one of these processes is allowed to start. From the hardware and control points of view, the VIS and NIR spectrographs are completely independent. This means that if there is a technical problem with one of the spectrographs, the other one is still fully functional and can be used for observations.

To facilitate the technical operation and maintenance of CARMENES for the observatory staff, the interlock system provides a process visualization user interface and powerful diagnostics that make all necessary information available in a clear graphical way. A touch screen shows a graphic view of the vacuum and climatic chambers, showing the state of each pump and valve and all relevant physical data. Temperature data at different points of the instrument and the vacuum pressure values are indicated in an intuitive way.

\subsection{Instrument Control System}

The instrument control system (ICS, Colomé et al. 2016) is implemented in Java and is based on JGoodies libraries. It provides an integrated environment for the operation of CARMENES. It is based on a modular architecture and a high level of abstraction design motivated by the heterogeneity of the different subsystems. A master/slave model architecture is used to build the control layer, where the ICS acts as a master and almost every other subsystem is a slave. The ICS acts as a slave only for the user interface subsystem, which controls and monitors the ICS functionalities. The instrument subsystems are abstracted into the ICS with a logic representation. Each subsystem is divided into specific functionalities, such as data representation, actions and events. The subsystem data are reported periodically to the ICS using its communication protocol and are stored into a central database, thus providing a snapshot of the system status.

The CARMENES graphical user interface (GUI) monitors and controls all instrument subsystems in normal operations. Other operation modes, such as the engineering control of all subsystems, are also supported by the GUI and are routinely used by the observatory staff for very specific commissioning or maintenance tasks.

Integrated into the ICS is a scheduler (CARMENES Scheduler Tool, CAST) that translates the survey strategy into a detailed observing schedule. It prepares a list of potential targets off-line, based on their visibility and on scientific priorities, and it contains an on-line module that suggests the "best" target to go to next after completion of each exposure.

\subsection{Data Reduction Pipeline}

The CARMENES pipeline receives the raw echelograms from both spectrographs, performs basic data reduction and the extraction of the spectra, and determines the radial velocity. The extraction pipeline CARACAL written in IDL is based on the REDUCE package (Piskunov \& Valenti 2002), but many parts have been modified and a number of new scripts have been added. The on-line version of CARACAL waits for new raw files, which are provided by the instrument control system at the end of each exposure, and performs basic processing (bias subtraction, flat fielding, cosmic ray correction, etc.), followed by a flat-relative optimal extraction of the 1D spectra (Zechmeister et al. 2014) and wavelength calibration.

The radial-velocity pipeline SERVAL offers different operation modes. Its main use is for the computation of high-precision radial velocities using a least-squares fitting algorithm. Here the observations are modeled with a template that is established from the observations themselves by a "shift and co-add" algorithm. AngladaEscudé \& Butler (2012) demonstrated that this approach can provide more precise radial velocities than the 

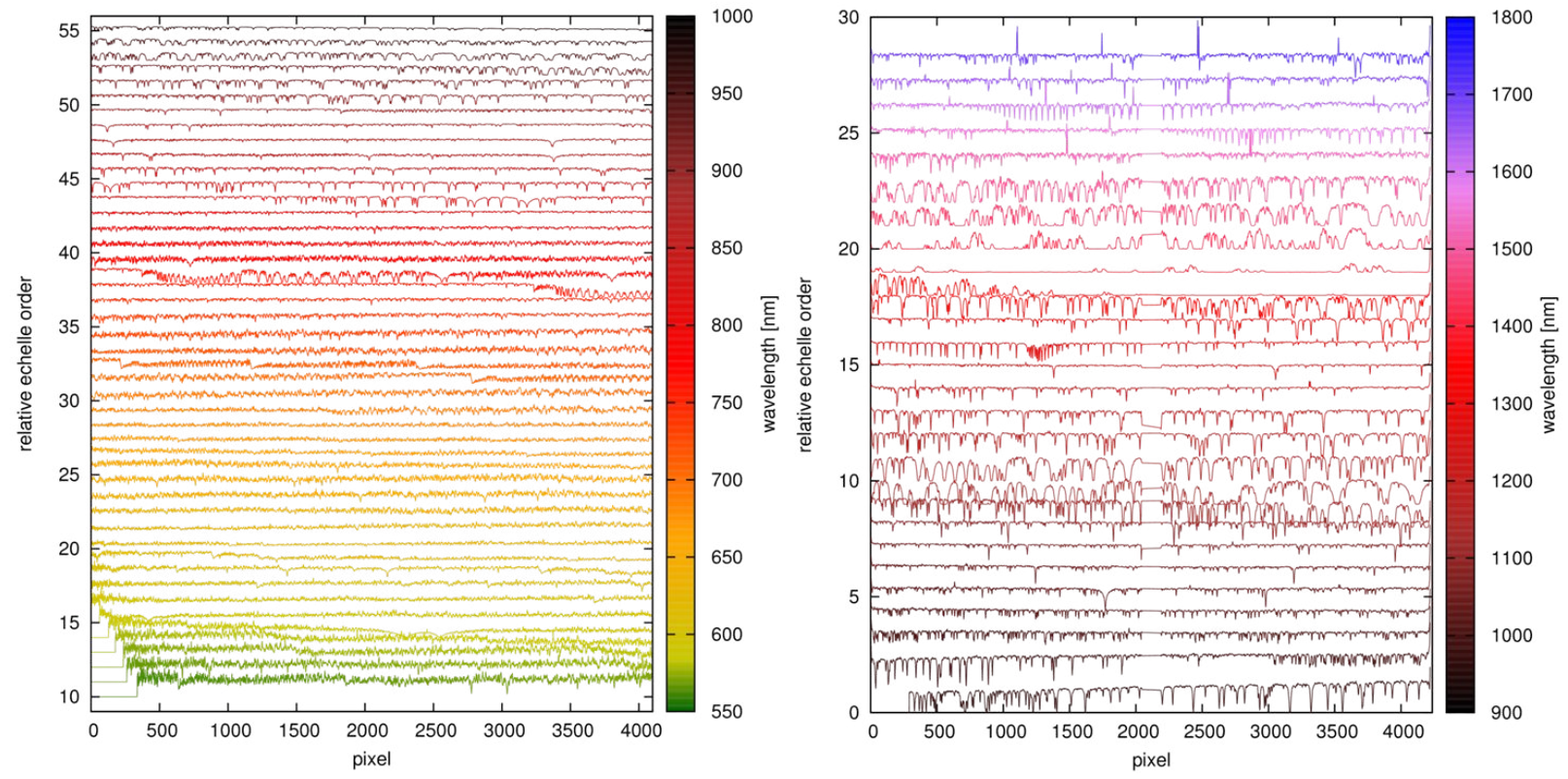

Figure 2. Full extracted spectrum showing stellar lines as well as telluric absorption and emission.

cross-correlation method. However, this mode is fully differential, i.e., the radial velocities have an arbitrary zero point. This radial-velocity pipeline also needs access to archived data for the computation of the template and is therefore normally used off-line.

\subsection{Data Flow and Archive}

The end-to-end data flow of the CARMENES survey proceeds through the following steps (see Caballero et al. 2016 for details): the CARMENES input catalogue (Carmencita), the scheduler (CAST), instrument control system and graphical user interface, the generation of raw data and fits headers, the spectrum extraction and wavelength-calibration pipeline (CARACAL), the observatory data repository and archive, the guaranteed time observations data archive, the radial-velocity pipeline (SERVAL), the data products archive (RADAR), and the visualization tool (CAVEMAN). This whole chain has been designed with the goal of high precision in mind, which means that calibration and environmental information is passed along with the data (e.g., in the FITS headers).

Each morning, the data files generated during the previous night are copied to a repository common to all instruments at the CAHA Observatory. From there, they can be retrieved by authorized users (usually the PI of open-time observing projects, or designated team members as in the case of the CARMENES M star survey). For the survey data, there is a second archive hosted by the Spanish Virtual Observatory in Madrid (CAB), which provides easy and reliable access to raw and processed data obtained during the guaranteed time observations. After expiration of the three-year proprietary period, the raw data and processed spectra from the CARMENES survey will be made publicly available through this portal.

\section{AIV AND COMMISSIONING}

In the spring of 2013, shortly after the preliminary design review of CARMENES, the agencies operating the CAHA observatory (i.e., the Max-Planck Society and the Consejo Superior de Investigaciones Científicas) declared that continued funding of CAHA was contingent upon readiness of the CARMENES instrument for scientific use by Dec 31, 2015. This decision put unusual pressure on the project management (García-Vargas et al. 2016), system engineering (Pérez-Calpena et al. 2016), and the spectrograph integration teams (Becerril et al. 2016a, Seifert et al. 2016). 

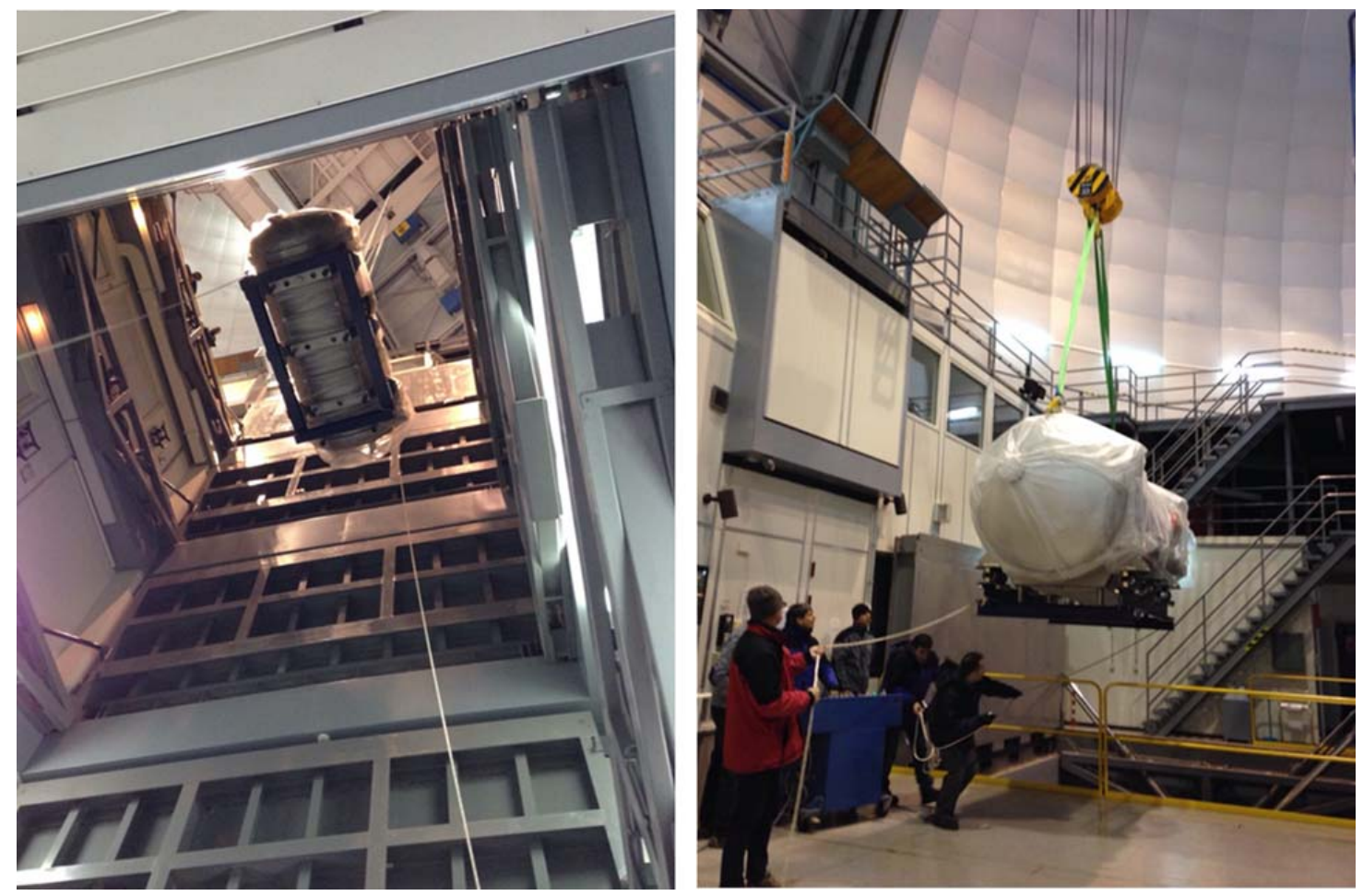

Figure 3. The near-infrared spectrograph of CARMENES was moved to Calar Alto Observatory and installed in the coudé laboratory of the $3.5 \mathrm{~m}$ telescope on Oct 20, 2015. The vacuum tank of the visible-light spectrograph is very similar; it was installed already in August.

\subsection{Assembly, Integration, and Verification (AIV)}

The subsystems of CARMENES were pre-integrated by several of the consortium member institutions: the front end, the fiber system, and the optical bench and detector cryostat of the visible spectrograph at the Landessternwarte Heidelberg, the VIS vacuum system and the NIR detector cryostat at the Max-Planck-Institut für Astronomie, the NIR spectrograph at the Instituto de Astrofísica de Andalucía, the calibration units at the Thüringer Landessternwarte Tautenburg, and the Fabry-Pérot etalons at the Institut für Astrophysik in Göttingen. The instrument control software development was led by the Institut de Ciències de l'Espai, and the electronics and software for the acquisition and guiding camera by the Hamburger Sternwarte. The CAHA observatory was responsible for refurbishing the coudé room of the $3.5 \mathrm{~m}$ telescope, site infrastructure, and the interlock system. A contract for management and system engineering support was given to FRACTAL SLNE. This distributed development reflects the diversity of funding sources for CARMENES (see acknowledgement below), with German and Spanish entities each contributing about half of the resources. It clearly posed challenges for the project management, but it also created flexibility with numerous interactions between the partners and contributions across institutional boundaries.

The first subsystems completed were the two calibration units, as these were needed for the integration of the two spectrographs. Therefore they were shipped to Heidelberg and Granada, respectively. The front end and fiber system were completely integrated and tested at the Landessternwarte; these components were the first to be sent to CAHA. The AIV plans of the two spectrographs were modified several times to save time in face of the strict delivery deadline. For the visible-light spectrograph the most drastic measure was the decision not to integrate the optical bench with the vacuum system in Heidelberg, but to perform this critical task only after shipping to the mountain. The near-IR spectrograph was fully integrated in Granada (including the detector cryostat supplied by the Max-Planck-Institute), but the number of cryo-vacuum-cycles was kept to an absolute minimum. There were thus several instances in which conscious decisions were taken to accept increased risk for schedule gains; in the end the AIV process was successful due to the dedication of a highly motivated team. 


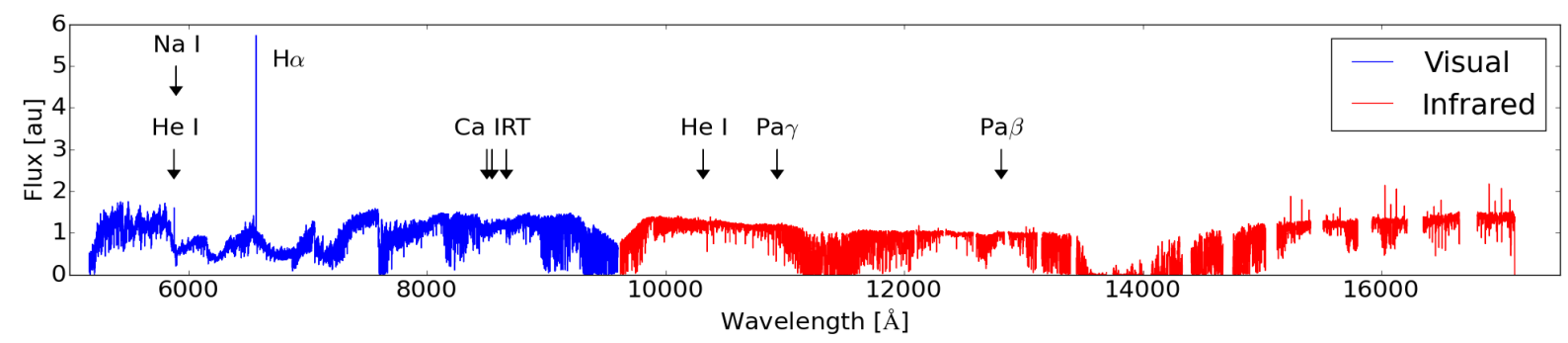

Figure 4. Complete visual (blue) and infrared (red) CARMENES spectrum of the active M4.5V dwarf YZ CMi (GJ 285); the spectrum is not flux calibrated. Chromospherically sensitive lines are distributed across the entire spectrum; a number of prominent examples are specifically indicated. In the optical wavelength range, the $\mathrm{H} \alpha$ line is particularly prominent and several molecular band heads can be distinguished. The infrared spectrum shows the atmospheric J- and H-band spectral windows around 12,000 and $16,000 \AA$. A number of airglow emission lines are prominent redward of $15,000 \AA$. For details see Czesla et al., contribution to the Cool Stars 19 conference (2016).

\subsection{Installation at CAHA}

All subsystems of CARMENES could thus be moved to Calar Alto and installed at the $3.5 \mathrm{~m}$ telescope in the course of 2015. The front end was mounted at the Cassegrain flange in April, followed by extensive testing of the acquisition and guiding procedures and the software interfaces with the telescope control system. The optical fibers connecting the front end to the spectrographs were routed through the telescope fork at the same time. The visible-light spectrograph was shipped to the observatory in July. The optical bench and the vacuum system had been separately pre-integrated at Landessternwarte Heidelberg and at the Max-Planck-Institut für Astronomie, respectively; they were first integrated with each other on site as explained above. The near-infrared spectrograph was fully integrated at the Instituto de Astrofísica de Andalucía and moved to Calar Alto in October (see Fig. 3). The calibration system and the Fabry-Pérot etalons were installed in parallel.

By staggering the arrival of the individual subsystems at the observatory, it was possible to distribute the work load on the observatory staff over several months, and to reduce the technical and schedule risks by performing subsystem tests in advance of the full instrument integration.

\subsection{First Light and Scientific Commissioning}

With all subsystems installed at their final locations at the $3.5 \mathrm{~m}$ telescope, CARMENES had "First Light" defined as taking stellar spectra with both spectrographs simultaneously - on Nov 9, 2015. This event marked the beginning of the scientific commissioning, in which the full instrument was tested and characterized through onsky observations. Since this phase was compressed into six weeks, the task of establishing the long-term stability of the spectrographs and radial-velocity measurements had to be deferred into the operational phase. Nevertheless, the functionality and operability of all subsystems could be verified, and CARMENES was provisionally accepted by CAHA on Dec 30, 2015.

During the final weeks of AIV and the first on-sky tests it became apparent that the original calibration strategy - taking simultaneous exposures of stars and hollow-cathode lamps with the two fibers of each spectrograph - was problematic because of the very large contrast between the brightest lines of the lamps and the numerous weak lines needed for calibration. The strategy was therefore modified to take simultaneous exposures of stars and the Fabry-Pérot etalons, and to tie the etalons to the hollow-cathode lamps with spectra taken during day time to ensure long-term stability.

\subsection{Initial Results}

The CARMENES M dwarf survey started on Jan 1, 2016. More than 200 distinct M dwarfs have been observed with the two channels during the first half year of science operations. As an example, Fig. 4 shows the complete visual and infrared CARMENES spectrum of the active M4.5V dwarf YZ CMi. Some important activity indicators are marked in this figure. Their variations in time series of spectra contain important information on the physical mechanisms of stellar activity, and will help to disentangle activity-induced radial-velocity variations 

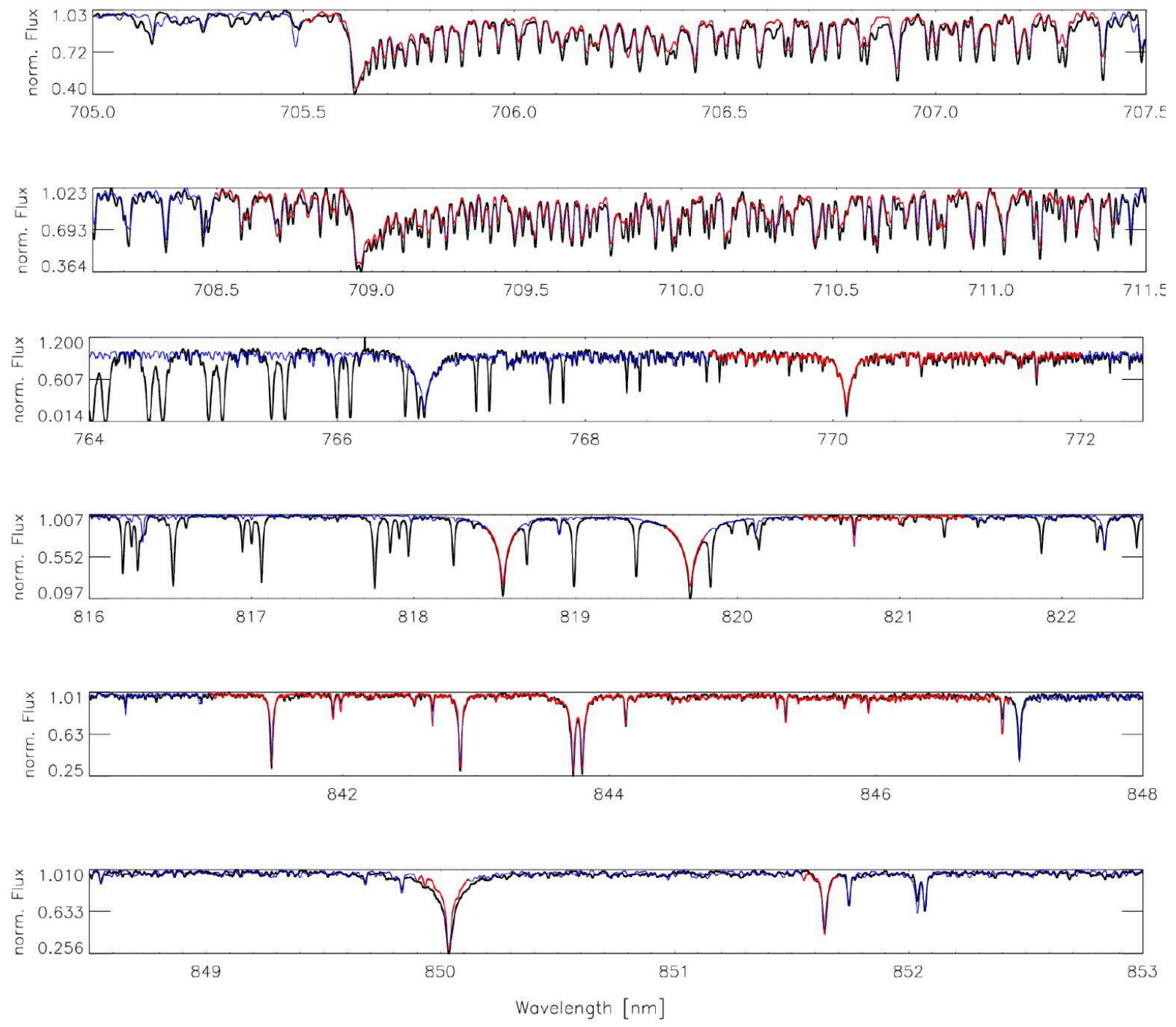

Figure 5. Sections of a CARMENES spectrum of the M1.5V star BD+44 2051 (GJ $412 \mathrm{~A}$, black) and the best fit model from a grid of PHOENIX-based model spectra (blue: model outside fit region, red: model inside fit regions for $\chi^{2}$-minimization). For details see Passegger et al., contribution to the Cool Stars 19 conference (2016).

Proc. of SPIE Vol. $9908990812-9$ 

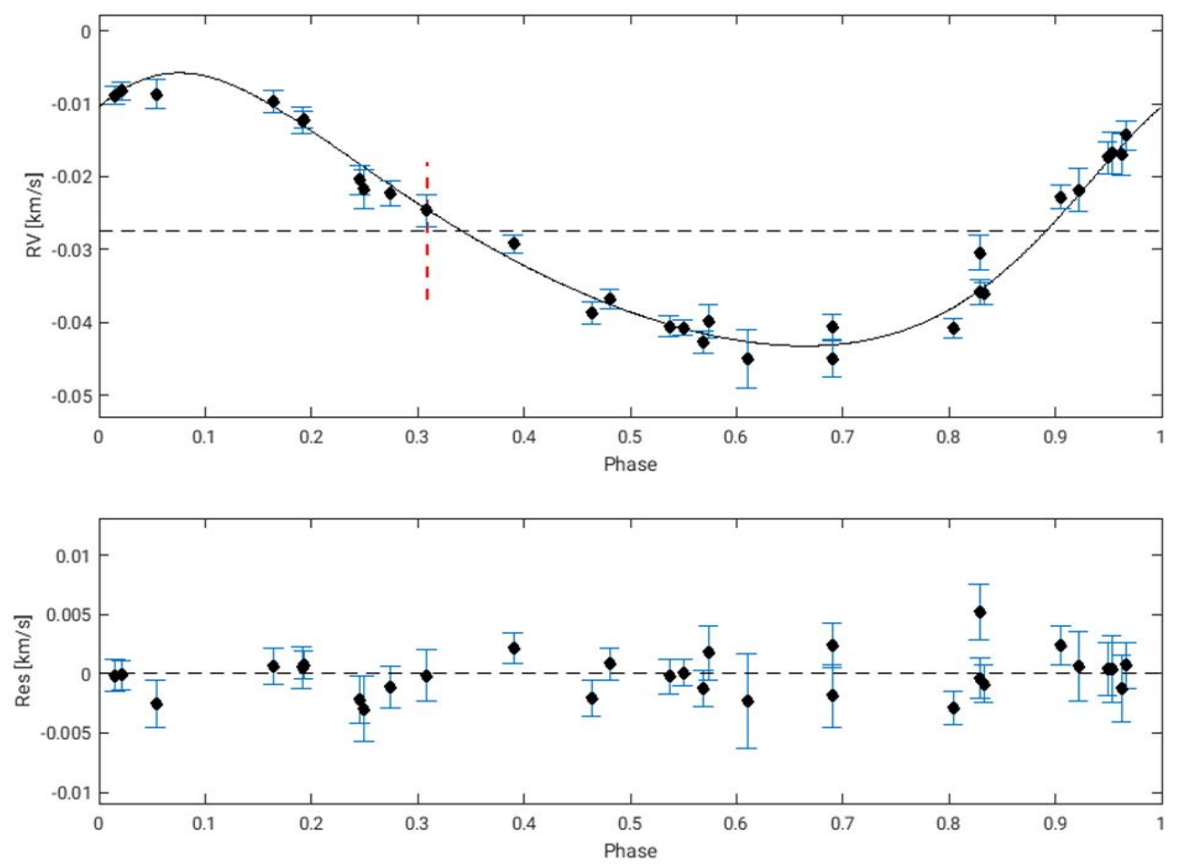

Figure 6. Radial velocities of the M2V star GJ 436 (Ross 905) collected with the visible-light spectrograph of CARMENES over a time span of $\sim 100 \mathrm{~d}$. The (previously known) planet orbiting this star induces a radial-velocity variation with a period of $\sim 2.6 \mathrm{~d}$ and a semi-amplitude of $\sim 18 \mathrm{~m} / \mathrm{s}$. The $\mathrm{rms}$ noise around the best Keplerian fit to our data is $\sim 2 \mathrm{~m} / \mathrm{s}$; this includes jitter due to stellar activity. The orbital parameters derived from this fit agree well with those by Lanotte et al. (2014).

from the signatures of planetary companions. In-depth studies of stellar activity and variability will be a valuable by-product of the CARMENES radial-velocity survey.

The small sections of the CARMENES spectrum of BD+44 2051 (spectral type M1.5V) displayed in Fig. 5 demonstrate the use of our high-resolution high-signal-to-noise data for detailed spectral analyses. A dedicated effort to improve current models of $\mathrm{M}$ star atmospheres will be required to take full advantage of the wealth of information provided by the CARMENES survey.

As part of the science verification we have observed a few M stars with known planetary companions. Radial velocities of GJ 436 (spectral type M2V) from the visible-light spectrograph of CARMENES are shown in Fig. 6. They were collected over a time span of $\sim 100$ days and have been folded with the orbital period of the companion $(\sim 2.6 \mathrm{~d})$. The semi-amplitude of the RV variations is $\sim 18 \mathrm{~m} / \mathrm{s}$. The rms noise around the best Keplerian fit to the visible-channel CARMENES data is $\sim 2 \mathrm{~m} / \mathrm{s}$ (including jitter due to intrinsic stellar variability), demonstrating the stability of the instrument and calibration procedures on the time scale of several months even in this early phase of operations. The analysis of the corresponding data from the NIR channel is still ongoing.

\section{THE CARMENES SURVEY}

\subsection{Survey Plans}

The most important scientific objective of CARMENES is to carry out a survey of late-type main sequence stars with the goals of characterizing the population of planets around these stars, and of detecting low-mass planets in their habitable zones (HZs). In the focus of the project are very cool stars with spectral type M4V or later and moderately active stars, but the target list also comprises earlier and therefore brighter M dwarfs. In particular, we aim at being able to detect a $2 \mathrm{M}_{\oplus}$ planet in the $\mathrm{HZ}$ of an M5V star. A long-term radial velocity precision of $1 \mathrm{~m} / \mathrm{s}$ per measurement $\left(1 \sigma_{i}\right)$ will permit to attain this goal. For stars later than $\mathrm{M} 4\left(M \lesssim 0.25 \mathrm{M}_{\odot}\right)$, 
such precision will yield detections of super-Earths of $5 \mathrm{M}_{\oplus}$ and smaller inside the entire width of the HZ. For a star near the hydrogen-burning limit, a planet with the mass of our own Earth in the HZ could be detected at a precision of $1 \mathrm{~m} / \mathrm{s}$. In addition, the $\mathrm{HZ}$ of all M-type dwarfs can be probed for super-Earths. Thus, a RV precision of $1 \mathrm{~m} / \mathrm{s}$ can trigger a breakthrough in exoplanet research in the spectral range of mid and late M-type stars.

We plan to survey a sample of $\sim 300$ M-type stars for low-mass planet companions. Besides the detection of the individual planets themselves, the ensemble of objects will provide sufficient statistics to assess the overall distribution of planets around $\mathrm{M}$ dwarfs: frequency, masses, and orbital parameters. The survey will confirm or falsify the seemingly low occurrence of Jovian planets around M stars, and the frequency of ice giants and terrestrial planets will be established along with their typical separations, eccentricities, multiplicities, and dynamics. Thus, the CARMENES survey can provide robust statements about planet formation in the low-mass star regime. It will complement ongoing and future transit surveys, which are more strongly biased towards short-period planets.

Additionally, the CARMENES planet search experiment will deliver valuable targets for further characterization, both their bulk and detailed properties. This is especially tantalizing for low-mass planets in the HZ, since determining their nature (e.g., icy or rocky) becomes feasible. Further, their study can potentially reveal the existence of an atmosphere and, perhaps, determine its chemical composition. The exoplanets from CARMENES will be prime objects for further characterization, via photometric modulation or direct detection, because most of the targets will be within 20 pc.

At least 600 clear nights have been allocated for the CARMENES Survey in the time frame from 2016 until 2018. This will allow us to obtain 60 observations on average for each of the 300 target stars, and more than 100 observations for selected objects. This is necessary to obtain secure detections and orbital parameters for planets with RV semi-amplitudes close to the measurement uncertainty.

\subsection{Science Preparation}

The selection of the target sample for the CARMENES M star survey is no trivial matter, since comprehensive, reliable and uniform information on the stellar properties is needed. We have therefore created a data based dubbed Carmencita (CARMEN[ES] Cool star Information and daTa Archive), collecting all available information on $\sim 2200$ bright M dwarfs accessible from Calar Alto (Quirrenbach et al. 2012, Caballero et al. 2013). For each star, we tabulate dozens of parameters: accurate astrometry, spectral typing, photometry in 20 bands from the ultraviolet to the mid-infrared, rotational and radial velocities, X-ray count rates and hardness ratios, close and wide multiplicity data and many more. This literature compilation was complemented by our own observing program, including high-resolution "lucky imaging" (Cortés-Contreras et al. 2015), low-resolution spectroscopy (Alonso-Floriano et al. 2015), and high-resolution spectroscopy. Altogether, Carmencita is probably the most comprehensive data base of nearby $\mathrm{M}$ dwarfs. It will be publicly released soon and then become a valuable resource for the whole community.

\subsection{Target Sample}

Since the scientific goals of CARMENES include collecting statistical information about the occurrence of planetary systems as a function of stellar mass in the regime $M \lesssim 0.6 \mathrm{M}_{\odot}$, the target sample should be based on well-defined selection criteria, so that potential selection biases can easily be understood and modeled. In addition, the targets should cover the spectral subtypes from M0 to late M as uniformly as possible. However, there is a tension of this requirement with the desire to give preference to the cooler subtypes, which is motivated by the larger radial-velocity signal of planets in their habitable zones, and by the stronger competitive advantage of CARMENES with respect to instruments working at shorter wavelengths. Further practical limitations are given by the small number of very cool stars that are sufficiently bright $J \lesssim 11.5$, and by the need to include a fair number of "poor weather" stars that can be observed under less than optimum conditions (e.g. cirrus, poor seeing).

Some stars are problematic for observations with CARMENES for technical reasons. This concerns mostly binaries with separations $\lesssim 5^{\prime \prime}$, in which the spectrum of the primary may be contaminated by the secondary. These stars have been flagged as "bad" in Carmencita, and are not considered viable targets. 


\begin{tabular}{|c|c|c|c|c|c|c|c|c|}
\hline & $\begin{array}{l}\text { M0 } \\
\mathrm{J}<7\end{array}$ & $\underset{\mathrm{J}<7.5}{\mathrm{M} 1}$ & $\begin{array}{l}M 2 \\
\mathrm{~J}<8\end{array}$ & $\begin{array}{c}\text { M3 } \\
\mathrm{J}<8.5\end{array}$ & $\begin{array}{l}\text { M4 } \\
J<9\end{array}$ & $\begin{array}{c}\text { M5 } \\
\text { J<9.5 }\end{array}$ & $\begin{array}{l}>\mathrm{M} 6 \\
\mathrm{~J}<10\end{array}$ & Total \\
\hline $4-5$ & 2 & 2 & & & & & & 4 \\
\hline $5-6$ & 6 & 6 & 1 & 3 & 1 & & & 17 \\
\hline $6-7$ & 20 & 19 & 13 & 9 & 6 & 1 & & 68 \\
\hline $7-8$ & & 18 & 37 & 47 & 13 & & 1 & 116 \\
\hline $8-9$ & & & & 46 & 73 & 9 & 3 & 131 \\
\hline $9-10$ & & & & & & 13 & 3 & 16 \\
\hline \multirow[t]{2}{*}{$10-12$} & & & & & & & 3 & 3 \\
\hline & 28 & 45 & 51 & 105 & 93 & 23 & 10 & 355 \\
\hline
\end{tabular}

Figure 7. Distribution of the target stars for the CARMENES survey as a function of M sub-type (horizontal axis) and stellar J magnitude (vertical axis).

With these considerations in mind, we have chosen to rank the "good" stars with declination $\delta>-23^{\circ}$ by apparent magnitude within each spectral subtype, and to select the brightest stars in each subclass. This creates a sliding magnitude cut-off that helps biasing the sample towards later spectral subtypes, while maintaining a simple selection criterion that can be modeled easily in statistical analyses. As can be seen from Fig. 7, the "sweet spot" for CARMENES is in the M3/M4 spectral range. Earlier M subtypes can be observed quite efficiently with spectrographs working at bluer wavelengths; reaching larger numbers of later stars requires near-IR spectrographs at larger telescopes.

\subsection{Future Prospects}

CARMENES is unique among current radial-velocity instruments in providing broad wavelength coverage from the green all the way to the near-infrared $\mathrm{H}$ band. It is thus very well suited for addressing the thorny issue of separating the Keplerian reflex motion induced by low-mass companions from stellar "noise" induced by photospheric processes. While current follow-up observations with near-IR spectrographs are already useful for confirming or refuting planet candidates found in the visible (e.g. Trifonov et al. 2015), CARMENES will take the next step by providing simultaneous coverage over a large wavelength range for all observed targets. This will eliminate difficulties due unequal and non-contemporaneous sampling of the radial-velocity curves in the visible and the near-IR.

The characteristics of CARMENES (red sensitivity, broad wavelength coverage, installation at a medium-size telescope well-suited for projects requiring large amounts of observing time) make this instrument very well suited also for follow-up of exoplanet searches with the transit method. After the completion of the CARMENES M star survey, the instrument could thus play a vital role in supporting TESS (Ricker et al. 2014) and later ESA's PLATO mission (Rauer et al. 2014). While a detailed scenario for the operation of CAHA and CARMENES in the next decade still has to be worked out, it is already evident that the long-term scientific prospects for the instrument are very good.

\section{ACKNOWLEDGMENTS}

CARMENES is an instrument for the Centro Astronómico Hispano-Alemán de Calar Alto (CAHA, Almería, Spain). CARMENES is funded by the German Max-Planck-Gesellschaft (MPG), the Spanish Consejo Superior de Investigaciones Científicas (CSIC), the European Union through FEDER/ERF funds, and the members of the CARMENES Consortium (see institutions ${ }^{\mathrm{a}}$ through ${ }^{\mathrm{k}}$ of the author list), with additional contributions by 
the Spanish Ministry of Economy, the state of Baden-Württemberg, the German Science Foundation (DFG), the Klaus Tschira Foundation (KTS), and by the Junta de Andalucía.

\section{REFERENCES}

[1] Alonso-Floriano, F.J., Morales, J.C., Caballero, J.A., Montes, D., Klutsch, A., et al. (2015). CARMENES input catalogue of $M$ dwarfs. I. Low-resolution spectroscopy with CAFOS. A\&A 577, A128

[2] Anglada-Escudé, G., \& Butler, R.P. (2012). The HARPS-TERRA Project. I. Description of the Algorithms, Performance, and New Measurements on a Few Remarkable Stars Observed by HARPS. ApJS 200, 15

[3] Becerril, S., Cárdenas, C., Amado, P., Abril, M., Ferro, I., et al. (2016a). CARMENES-NIR channel spectrograph: how to achieve the full AIV at system level of a cryo-instrument in nine months. These proceedings, 9910-32

[4] Becerril, S., Mirabet, E., Lizon, J.L., Abril, M., Cárdenas, C., et al. (2016b). CARMENES-NIR channel spectrograph - Cooling system AIV: Thermo-mechanical performance of the instrument. These proceedings, 9912-208

[5] Caballero, J.A., Cortés-Contreras, M., López-Santiago, J., Alonso-Floriano, F.J., Klutsch, A., et al. (2013). CARMENES. III. CARMENCITA, the input catalogue. In Highlights of Spanish astrophysics VII, proceedings of the X scientific meeting of the Spanish Astronomical Society (SEA). Eds. Guirado, J.C., Lara, L.M., Quilis, V., \& Gorgas, J., p. 645

[6] Caballero, J.A., Guàrdia, J., López del Fresno, M., Zechmeister, M., de Juan, E., et al. (2016). CARMENES: data flow. These proceedings, 9910-98

[7] Colomé, J., Guàrdia, J., Hagen, H.J., Morales Muñoz, R., Abril, M., et al. (2016). CARMENES: The CARMENES instrument control software suite. These proceedings, 9913-149

[8] Cortés Contreras, M., Caballero, J.A., Bejar, V.J.S., Gauza, B., Montes, D., et al. (2015). Preparation of the CARMENES input catalogue: Multiplicity of $M$ dwarfs from tenths of arcseconds to hundreds of arcminutes. In $18^{\text {th }}$ Cambridge workshop on cool stars, stellar systems and the Sun. Eds. van Belle, G., \& Harris, H.C., p. 805

[9] Fischer, D.A., Anglada-Escudé, G., Arriagada, P., Baluev, R.V., Bean, J.L., et al. (2016). State of the field: extreme precision radial velocities. PASP 128, 066001

[10] García-Vargas, M.L., Caballero, J., Pérez-Calpena, A., Amado, P., Seifert, W., et al. (2016). CARMENES: management of a schedule-driven project. These proceedings, 9911-24

[11] Helmling, J., Wagner, K., Hernández Castaño, L., Benítez, D., Marín Molina, J., et al. (2016). CARMENES: Interlocks or the importance of process visualization and system diagnostics in complex astronomical instruments. These proceedings, 9908-237

[12] Lanotte, A.A., Gillon, M., Demory, B.O., Fortney, J.J., Astudillo, N., et al. (2014). A global analysis of Spitzer and new HARPS data confirms the loneliness and metal-richness of GJ $436 \mathrm{~b}$. A\&A 572, A73

[13] Pérez-Calpena, A., Seifert, W., Amado, P., Quirrenbach, A., García-Vargas, M.L., et al. (2016). CARMENES. System engineering during manufacturing and AIV phases. These proceedings, 9911-78

[14] Piskunov, N.E., \& Valenti, J.A. (2002). New algorithms for reducing cross-dispersed echelle spectra. A\&A 385, 1095

[15] Quirrenbach, A., Amado, P.J., Mandel, H., Caballero, J.A., Mundt, R., et al. (2010). CARMENES: Calar Alto high-Resolution search for $M$ dwarfs with Exo-earths with Near-infrared and optical Echelle Spectrographs. In Ground-based and airborne instrumentation for astronomy III. Eds. McLean, I.S., Ramsay, S.K., \& Takami, H., SPIE 773513

[16] Quirrenbach, A., Amado, P.J., Seifert, W., Sánchez Carrasco, M.A., Mandel, H., et al. (2012). CARMENES. I: instrument and survey overview. In Ground-based and airborne instrumentation for astronomy IV. Eds. McLean, I.S., Ramsay, S.K., \& Takami, H., SPIE 84460R

[17] Quirrenbach, A., Amado, P.J., Caballero, J.A., Mundt, R., Reiners, A., et al. (2014). CARMENES instrument overview. In Ground-based and airborne instrumentation for astronomy V. Eds. Ramsay, S.K., McLean, I.S., \& Takami, H., SPIE 91471F

[18] Rauer, H., Catala, C., Aerts, C., Appourchaux, T., Benz, W., et al. (2014). The PLATO 2.0 mission. Exp. Astron. 38, 249 
[19] Ricker, G.R., Winn, J.N., Vanderspek, R., Latham, D.W., Bakos, G.Á., et al. (2014). Transiting Exoplanet Survey Satellite. JATIS 1, 014003

[20] Roth, M.M., Kelz, A., Fechner, T., Hahn, T., Bauer, S.M., et al. (2005). PMAS: The Potsdam MultiAperture Spectrophotometer. I. Design, Manufacture, and Performance. PASP 117, 620

[21] Seifert, W., Sánchez-Carrasco, M., Xu, W., Cárdenas, C., Sánchez-Blanco, E., et al. (2012). CARMENES. II: optical and opto-mechanical design. In Ground-based and airborne instrumentation for astronomy IV. Eds. McLean, I.S., Ramsay, S.K., \& Takami, H., SPIE 844633

[22] Seifert, W., Xu, W., Stahl, O., Hagen, H.J., Sánchez Carrasco, M.A., et al. (2016). CARMENES: The VIS channel spectrograph in operation. These proceedings, 9908-231

[23] Stürmer, J., Stahl, O., Schwab C., Seifert, W., Quirrenbach, A., et al. (2014). CARMENES in SPIE 2014. Building a fibre link for CARMENES. In Advances in optical and mechanical technologies for telescopes and instrumentation. Eds. Navarro, R., Cunningham, C.R., \& Barto, A.A., SPIE 915152

[24] Trifonov, T., Reffert, S., Zechmeister, M., Reiners, A., \& Quirrenbach, A. (2015). Precise radial velocities of giant stars. VIII. Testing for the presence of planets with CRIRES infrared radial velocities. A\&A 582, A54

[25] Zechmeister, M., Anglada-Escudé, G., \& Reiners, A. (2014). Flat-relative optimal extraction. A quick and efficient algorithm for stabilised spectrographs. A\&A 561, A59 